EPJ Web of Conferences 59, 17005 (2013)

DOI: $10.1051 /$ epjconf/20135917005

(C) Owned by the British Crown, published by EDP Sciences, 2013

\title{
Modelling hot electron generation in short pulse target heating experiments
}

\author{
N.J. Sircombe ${ }^{1,2}$ and S.J. Hughes ${ }^{1}$ \\ ${ }_{1}^{1}$ AWE, Aldermaston, Reading, Berkshire RG7 4PR, UK \\ 2 Centre for Fusion Space and Astrophysics, University of Warwick, Coventry CV4 7AL, UK
}

\begin{abstract}
Target heating experiments planned for the Orion laser facility, and electron beam driven fast ignition schemes, rely on the interaction of a short pulse high intensity laser with dense material to generate a flux of energetic electrons. It is essential that the characteristics of this electron source are well known in order to inform transport models in radiation hydrodynamics codes and allow effective evaluation of experimental results and forward modelling of future campaigns. We present results obtained with the particle in cell (PIC) code EPOCH for realistic target and laser parameters, including first and second harmonic light. The hot electron distributions are characterised and their implications for onward transport and target heating are considered with the aid of the Monte-Carlo transport code THOR.
\end{abstract}

\section{INTRODUCTION}

The Orion laser facility at AWE combines two petawatt class short-pulse (SP) beamlines and ten longpulse (LP) beams, each with a maximum energy of the order of 500 J. Short-pulse lasers allow material, which can be pre-compressed by the action of the long-pulse beams, to be heated to high temperatures before hydrodynamic motion can disassemble the target. This means that future Orion experiments will be able to study material properties across a range of temperatures and densities.

Modelling the disparate timescales involved in experiments combining both long and short pulse beams, such as those planned for the Orion laser facility, and many fast ignition schemes [1] presents a considerable challenge. Tackling such problems with a single code is unfeasible with existing compute resources. Instead, modern hydro-codes, incorporating advanced transport models utilising hot electron spectra obtained from Particle In Cell (PIC) models offer a practical route to integrated modelling.

We present results obtained with the PIC code EPOCH [2] for realistic target and laser parameters. The hot electron distributions generated in EPOCH are linked directly to the Monte Carlo transport code THOR, based on the approach detailed in Ref. [3], which models the target heating at solid densities and above [4]. These results are compared with equivalent simulations which use an electron spectrum derived from the well known ponderomotive scaling and it is demonstrated that the differences between the EPOCH spectra and simple 'single temperature' distributions have implications for onward transport and target heating.

\section{PIC MODELLING OF HOT ELECTRON GENERATION}

The 2D, relativistic PIC code EPOCH was used to model the interaction of both first (1.06 micron, "red") and second ( 0.53 micron, "green") harmonic light with a preformed deuterium plasma target. Peak electron density in the PIC simulations was limited to $3 \times 10^{29} \mathrm{~m}^{-3}$. In the case of first harmonic light it was assumed that the laser pre-pulse creates an exponential pre-plasma with a scale length of

This is an Open Access article distributed under the terms of the Creative Commons Attribution License 2.0, which permits unrestricted use, distribution, and reproduction in any medium, provided the original work is properly cited. 

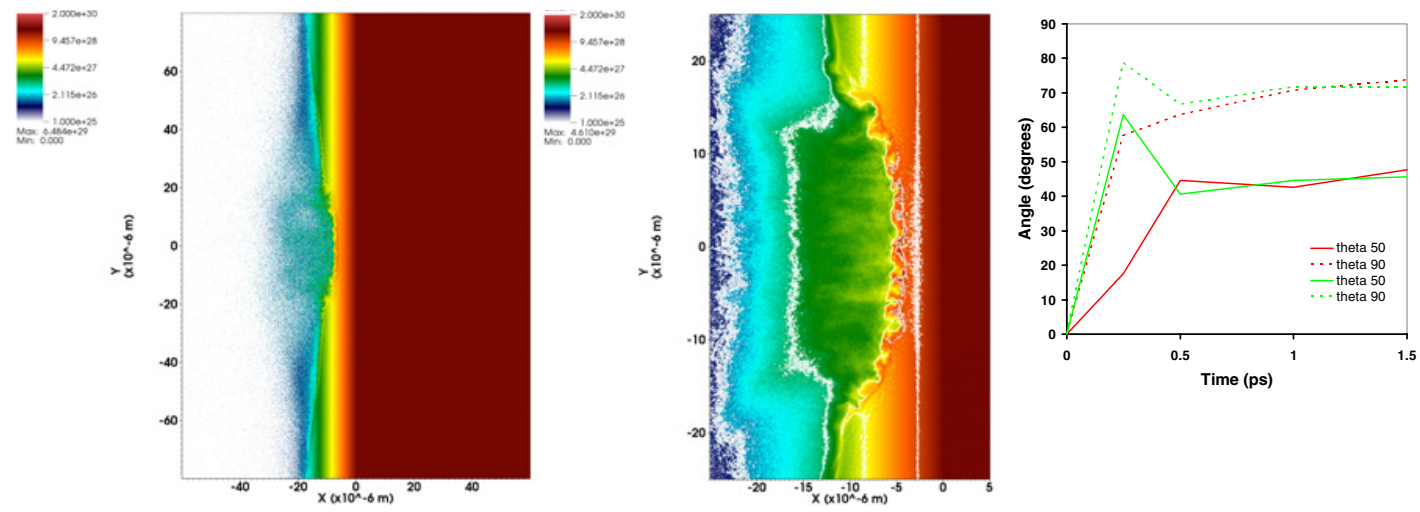

Figure 1. Electron density profile for the case of a first harmonic, high intensity $\left(3 \times 10^{20} \mathrm{Wcm}^{-2}\right)$ pulse interacting with a 2.5 micron scale-length pre-formed plasma after $1 \mathrm{ps}$ (left). Closer inspection of the interaction region after 0.5 ps (centre) with $0.1,1,10$ and 100 times critical density contours plotted in white displays clear "scalloping" of the critical density surface mid-way through the pulse. This is driven by laser filamentation in the pre-plasma, and is not present in the cases with minimal pre-plasma. It does not appear to increase the divergence of the hot electrons generated, as shown in plots of $\theta_{50}$ and $\theta_{90}$, the half-angle required to enclose $50 \%$ and $90 \%$ of the hot electron population respectively (right). Here red lines represent the first harmonic case, and green the second harmonic case.
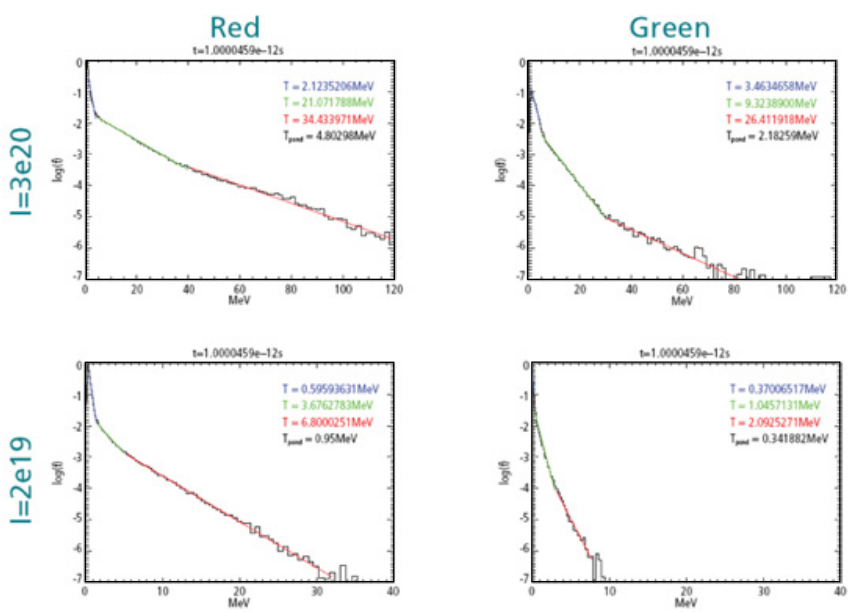

Figure 2. Time integrated hot electron spectra for high $\left(3 \times 10^{20} \mathrm{Wcm}^{-2}\right)$ and low $\left(2 \times 10^{19} \mathrm{Wcm}^{-2}\right)$ intensity runs, with first (red) and second (green) harmonic light. The resulting electron distribution is poorly characterised by a single "temperature" fit, particularly in the first harmonic case.

2.5 microns. In the second harmonic case, where a higher contrast would be expected, a 0.25 micron scale length was adopted.

The system size was 120 microns in $x$ by 160 microns in $y$ with a mesh resolution of $4800 \times$ 6400 cells, 96 particles per cell and thermal plasma boundaries. The laser profile was a second order supergaussian with a uniform temporal profile, following a $50 \mathrm{fs}$ linear ramp. Runs at intensities of $3 \times 10^{20} \mathrm{Wcm}^{-2}$ and $2 \times 10^{19} \mathrm{Wcm}^{-2}$ were conducted.

Figure 1 shows the electron densities at late time for the case of high intensity, first harmonic light. The "scalloping" of the critical density surface in the red case appears to be the result of laser 

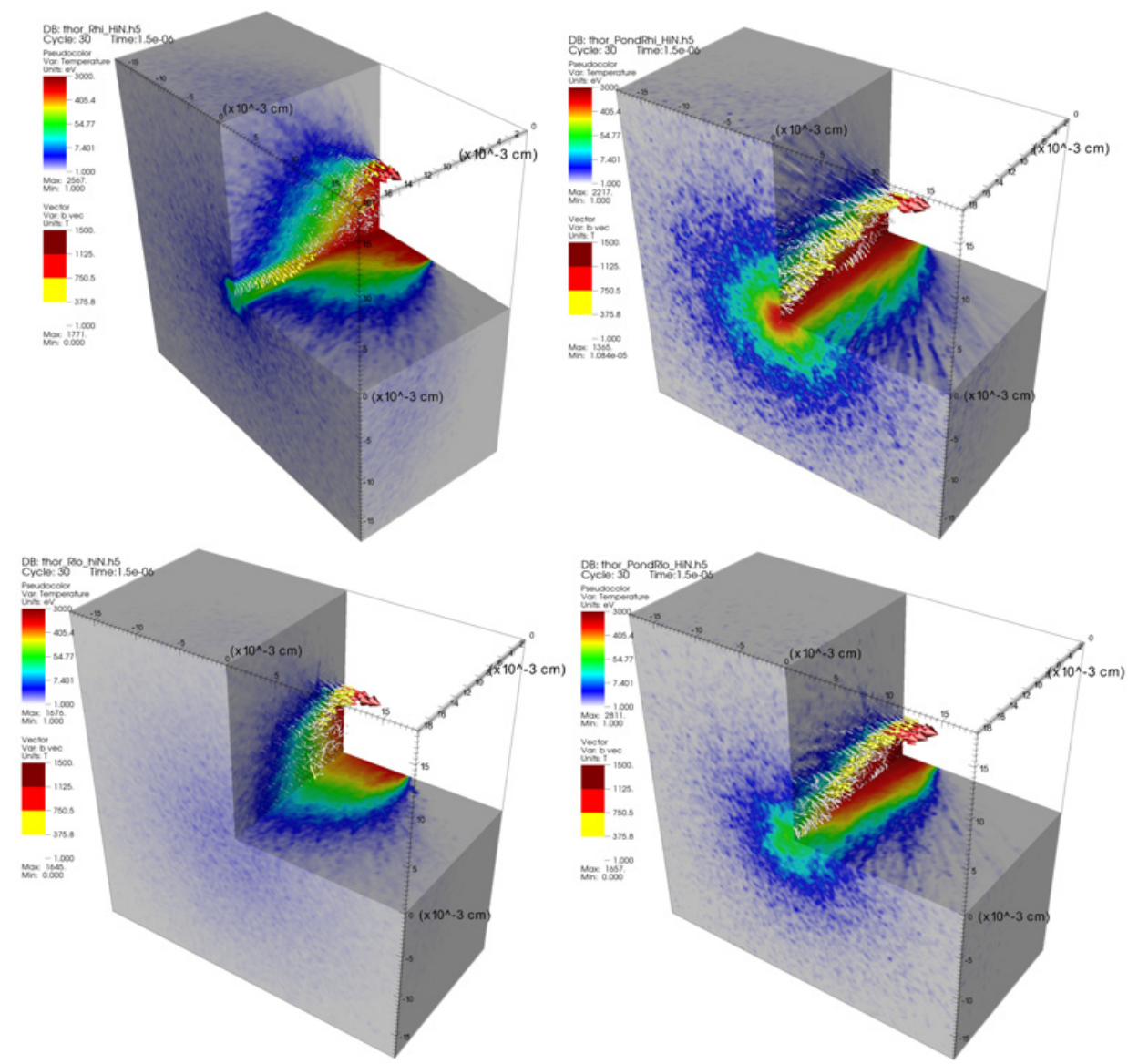

Figure 3. Material temperature profiles and B field vectors after $1.5 \mathrm{ps}$ for EPOCH (left) and ponderomotive (right) electron sources at high (top) and low (bottom) intensity red light. The EPOCH generated electron distribution produces a significantly different heating profile compared with the ponderomotive distribution in both cases reducing the heating at depth but enhancing heating near the surface.

filamentation seeded by the transient interference of an incident laser and its backscattered SRS daughter wave [5]. This process can only occur in the presence of a pre-plasma, and so, is absent in the second harmonic case. This substantial distortion to the critical surface does not appear to have a marked impact on the divergence of the hot electron population.

\section{LINKING PIC ABSORPTION AND MC TRANSPORT MODELS}

A particle probe diagnostic was placed a few microns behind peak density, to record the properties of each hot electron as it passes the probe plane. As shown in Figure 2 the hot electron spectra are poorly represented by a single hot "temperature", and we find the divergence angle to be weakly energy dependent with the higher energy component displaying some collimation. Therefore, rather than use a Boltzmann distribution for the hot electron source in our transport modelling, we sample the electron distribution captured by EPOCH's particle probe. Raw probe data is integrated onto a fixed mesh using a link code, LOKI, to produce an electron phase-space distribution in $(\mathrm{r}, \mathrm{E}, \theta)$, varying in time. 


\section{EPJ Web of Conferences}

\section{MC MODELLING OF HOT ELECTRON TRANSPORT}

A source term for the transport code THOR was generated by sampling the particle distribution functions generated from EPOCH probe data by the link code LOKI. This ensures the hot electrons in our transport model are consistent with those generated in our absorption model.

THOR is an explicit, hybrid electron transport code, with a Monte-Carlo VFP solver for the hot electron population. Various EOS and resistivity models available, ionisation effects are included and it can be linked to the 2D hydrocode CORVUS. For these simulations we used tabular EOS, a Spitzer resistivity model (capped at temperatures below $100 \mathrm{eV}$ ) and a Thomas-Fermi [6] based ionisation model. THOR was run in a 3D Eulerian configuration with a uniform density of deuterium of $10 \mathrm{~g} / \mathrm{cc}$, and a system size of $360 \times 360 \times 180$ microns. In Figure 3 we compare the heating profiles generated by the EPOCH source with those generated by a single temperature hot electron source given by a temperature from the normal ponderomotive scaling [7] with an absorption fraction total energy to match the equivalent EPOCH simulation. We observe a marked difference in the heating profiles. Heating from the EPOCH source is dominated by large numbers of lower energy electrons which loose energy via Ohmic heating and stop in the plasma. This difference is less pronounced in the low intensity second harmonic case, for which the ponderomotive temperature fit was less erroneous.

\section{SUMMARY}

Designing and evaluating experiments on modern laser systems such as Orion, or developing a fastignition point design requires a modelling capability which spans the disparate scales of both long-pulse and short-pulse laser-plasma interaction. Tackling such problems with a single code is clearly unfeasible with existing HPC resources. Instead, modern hydro-codes, drawing on advanced transport models populated with hot electron spectra obtained from fully kinetic codes will offer a practical route to fullyintegrated modelling. Here we have presented results where the source terms in our transport models are derived directly from our kinetic simulations. The resulting target heating profiles are substantially affected by the difference in electron spectra - in particular the energy deposited by the lower-energy components.

This clearly demonstrates the potential importance of correctly characterising the hot electrons generated by the laser-target interaction when considering onward transport and target heating. This is significant for the HEDP experiments planned for Orion as well as electron-driven Fast Ignition schemes. EPOCH can be linked to ALE and Lagrangian hydrocodes to provide initial plasma conditions, and THOR can be coupled with hydrocodes directly. Together with the EPOCH-THOR link described here, this provides an integrated modelling capability for combined LP / SP experiments. Future work will look to developing and exploiting this capability to support experiments on Orion.

\section{References}

[1] M. Tabak et al., Phys. Plasmas 1, 1626 (1994)

[2] http://www.ccpp.ac.uk

[3] J. R. Davies, A. R. Bell, and M. G. Haines, Phys. Rev. E 56, 7193 (1997)

[4] C. R. D. Brown et al., Phys. Rev. Lett 106, 185003 (2011)

[5] C. S. Brady, A. Lawrence-Douglas and T. D. Arber., Phys. Plasmas 19, 063112 (2012)

[6] M. N. Rosenbluth and R. Z. Sagdeev, Handbook of Plasma Physics, Volume 3, Physics of Laser Plasma (North-Holland, 1991)

[7] S. C. Wilks et al., Phys. Rev. Lett. 69, 1383, (1992) 